\title{
Review Article \\ Properties of the HiI Regions Derived Using Integral Field Spectroscopy
}

\author{
Sebastian F. Sánchez ${ }^{1,2,3}$ \\ ${ }^{1}$ Instituto de Astrofísica de Andalucía (CSIC), Glorieta de la Astronomía s/n, Apartado 3004, 18080 Granada, Spain \\ ${ }^{2}$ Centro Astronómico Hispano Alemán, Calar Alto (CSIC-MPG), C/Jesús Durbán Remón 2-2, 04004 Almería, Spain \\ ${ }^{3}$ Astronomical Institute, Academy of Sciences of the Czech Republic, Boční II 1401/1a, 14100 Prague, Czech Republic
}

Correspondence should be addressed to Sebastian F. Sánchez; sanchez@caha.es

Received 15 August 2013; Accepted 4 November 2013

Academic Editor: Polychronis Papaderos

Copyright (c) 2013 Sebastian F. Sánchez. This is an open access article distributed under the Creative Commons Attribution License, which permits unrestricted use, distribution, and reproduction in any medium, provided the original work is properly cited.

Here we review some of our more recent results on the observed properties of HiI regions using Integral Field Spectroscopy. In particular, we illustrate the use of this technique to study in detail the ionization conditions across the nebulae for galactic HiI regions (focused on the Orion Nebula) and the statistical study of large samples of extragalactic Hir regions. We review the reported new scaling relation between the local mass density and the oxygen abundance across the disk galaxies and the recently discovered universal gradient for oxygen abundances. We update our previous results the lack of a dependence of the Mass-Metallicity relation with the starformation rate, including new unpublished data. Finally we discuss on the relation between the ionization conditions in the nebulae and the underlying stellar population. All together our results indicate that disk galaxies present a chemical enrichment dominated by an inside-out growth scenario, with a less evident effect of radial migrations and/or outflows.

\section{Introduction}

Nebular emission lines from bright-individual His regions have been, historically, the main tool at our disposal for the direct measurement of the gas-phase abundance at discrete spatial positions in galaxies. A good observational understanding of the distribution of element abundances across the surface of nearby galaxies is necessary to place constraints on theories of galactic chemical evolution. The same information is crucial to derive accurate star formation histories of and obtain information on the stellar nucleosynthesis in normal spiral galaxies.

Several factors dictate the chemical evolution in a galaxy, including the primordial composition, the content and distribution of molecular and neutral gas, the star formation history (SFH), feedback, the transport and mixing of gas, the initial mass function (IMF), (e.g., $[1,2]$ and references therein). All these ingredients contribute through a complex process to the evolutionary histories of the stars and the galaxies in general. Accurate measurements of the present chemical abundance constrain the different possible evolutionary scenarios, and therefore it is important to determine the elemental composition using a common approch, among different galaxy types.

Previous spectroscopic studies have unveiled some aspects of the complex processes at play between the chemical abundances of galaxies and their physical properties. Although these studies have been successful in determining important relationships, scaling laws and systematic patterns (e.g., luminosity-metallicity, mass-metallicity, and surface brightness versus metallicity relations [3-7]; effective yield versus luminosity and circular velocity relations [8]; abundance gradients and the effective radius of disks [9]; systematic differences in the gas-phase abundance gradients between normal and barred spirals $[6,10]$; characteristic versus integrated abundances [11], etc.), they have been limited by statistics, either in the number of observed HiI regions or in the coverage of these regions across the galaxy surface.

The advent of Multiobject Spectrometers and Integral Field Spectroscopy (IFS) instruments with large fields of view 
now offers us the opportunity to undertake a new generation of emission-line surveys, based on samples of hundreds of HiI regions and full two-dimensional (2D) coverage of the discs of nearby spiral galaxies (e.g., [12-14]). On the other hand, these new techniques allow us to study with unprecedent detail the ionization conditions and chemical enrichment processes within the individual nebulae in the Galaxy and provide with unique links between those local processes and the global ones that govern the evolution of galaxies (e.g., $[15,16])$.

In the last few years we started a major observational program to understand the statistical and individual properties of Hir regions and to unveil the nature of the reported physical relations, using IFS. Here we present a summary of our main results, including the analysis of the spatially resolved ionization condition in the Orion Nebula (Section 2), the connection between the ionization conditions in the HII regions and the properties of the underlying stellar population (Section 3), some of the highlights of our current IFS studies (Section 4), and the evidence of an inside-out growth in disk galaxies on the basis that we have found (Section 5).

\section{The Orion Nebula}

The Orion Nebula is the brightest and best studied Hir region in the sky. It has been used for decades as a fundamental laboratory in the study of the star formation regions, the ionization processes, and the helium and heavy elements enrichment. However, despite the large number of studies on this target, there is still a lot to know about it, even from optical studies. The more we know the more complex it seems to be.

Several spectroscopic surveys have tried to characterize its spectroscopic properties, taken spectra at different "representative" locations (e.g., [17, 18]). In particular, Osterbrock [19] show a compilation of high and low dispersion deep spectra of the central bright region in the optical-NIR region (3000-11000 §).They measured 225 emission lines, 88 at the wavelength range of our IFS data. Using the relative intensities of these lines they derived the relative abundances of several elements, the electronic temperature $(T=9000 \mathrm{~K})$ and density $\left(N_{\mathrm{e}}=4 \times 10^{3} \mathrm{~cm}^{-3}\right)$, and the extinction $\left(A_{V} \sim\right.$ $1.08 \mathrm{mag}$, derived from the $\mathrm{H} \alpha / \mathrm{H} \beta$ ratio). Such properties are frequently compared with those of distant His regions in our Galaxy and extragalactic ones (e.g., [20]). However, due to their distance most of these regions are poorly resolved, and their integrated properties are compared with those of particular areas in the Orion Nebula.

Very little effort has been done to study the distribution of the physical properties across the Nebula, apart from some peculiar areas (e.g., [18]), or their integrated values in large aperture areas. Pogge et al. [21] performed FabryPerot (FP) imaging spectrophotometry on an area of $\sim 6^{\prime} \times 6^{\prime}$ centered in the Trapezium area. Their FP data covered the brightest emission lines in the Nebula $\mathrm{H} \beta$, [OIII] $\lambda 5007, \mathrm{H} \alpha$, $[\mathrm{NII}] \lambda 6548,6583,[\mathrm{SII}] \lambda 6716,6731$, and [HeI] $\lambda 6678$. They derived some of the average spectroscopic parameters of the core of the Nebula, including the integrated $\mathrm{H} \alpha$ flux, the average extinction, the average ionization line ratios $([\mathrm{OIII}] / \mathrm{H} \beta$,
$[\mathrm{NII}] / \mathrm{H} \alpha$ and $[\mathrm{SII}] / \mathrm{H} \alpha$ ), the $\mathrm{He}^{+} / \mathrm{H}^{+}$ionic abundance ratio, and the average electron density. Evenmore, they derived the first reliable maps of the distribution of these properties across the Nebula, which show a considerable degree of structure. They found significant differences with some of the previously reported values that correspond to particular regions in the Nebula (e.g., [19]). However, due to the reduced spectroscopic coverage of their dataset, it was not possible to fully characterize the ionization conditions in the Nebula.

In [15], we presented a low-resolution Integral Field Spectroscopy (IFS) survey of an almost similar field of view of the FP observations presented by Pogge et al. [21] but covering the entire optical wavelength range ( 3700-7100 $\AA)$. The data, comprising 8121 individual spectra sampling circular areas of $2.7^{\prime \prime}$ each one, were reduced to be released publicly for the community (http://www.caha.es/sanchez/orion/). This is the largest spectroscopic survey ever performed for a single HII region.

Line intensity maps for different emission lines were obtained to study the complex structure of the ionized gas in the Nebula. The derived $\mathrm{H} \alpha$ intensity map, shown in Figure 1(a), a tracer of the ionized hydrogen, was found to be similar to that one found using Fabry-Perot and narrowband imaging. The electron density and temperature maps were derived using the [SII] line ratios and [OIII] and [NII] line ratios, respectively, as described in [15]. A considerable amount of structure was found in both distributions. We used them to create a map of the expected $\mathrm{H} \alpha / \mathrm{H} \beta$ line ratio, based on case $\mathrm{B}$ recombination. A comparison between this expected line ratio and the observed one, that we show in Figure 1(b), was used to obtain a dust extinction map, that was used to correct all the derived emission lines for reddening.

The ionized structure was studied based on the classical diagnostic line ratios $[\mathrm{NII}] / \mathrm{H} \alpha$ and $[\mathrm{OIII}] / \mathrm{H} \beta$, shown in Figures $1(\mathrm{c})$ and $1(\mathrm{~d})$. The distribution of helium and oxygen abundances was derived based on the [HeI] $\lambda 6678 / \mathrm{H} \alpha$ line ratio and the temperature metallicity indicator (based on the $[\mathrm{OII}] / \mathrm{H} \beta$ and $[\mathrm{OIII}] / \mathrm{H} \beta$ line ratios). No clear relation was found between both abundance distributions, contrary to expected from the study of the integrated properties of known HiI regions. There is a relation between the helium abundance and the ionization structure, in the sense that partially ionized areas are less abundant in helium and more abundant in heavy elements. Two possible deviations from case B recombination theory in the Orion Nebula were proposed by [15] to explain this trend and the lack of relation between the Helium and Oxygen abundances: (i) the fact that the Nebula is not fully ionized, which could lead to a systematic underestimation the helium mass fraction due to the nonnegligible contribution of neutral helium, and (ii) the suspicion that there are dust grains mixed with the emitting gas which absorbs the ionizing Lyman-continuum photons and not a simple dust screen.

The oxygen abundance presents a rich structure that it is not clearly related to the ionization structure. At a first order it has positive gradient, with a minimum near the trapezium (the ionizing source), of the order of $12+\log (\mathrm{O} / \mathrm{H}) \sim 8.2$, increasing towards the outer parts, where it reaches a level of $12+\log (\mathrm{O} / \mathrm{H}) \sim 8.8$. This is a good example of how 


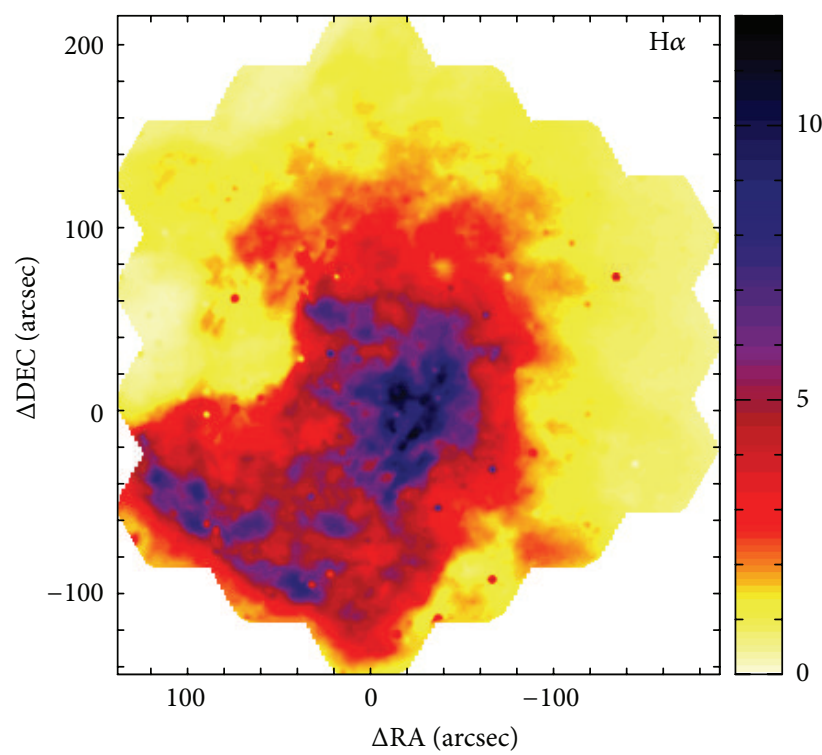

(a)

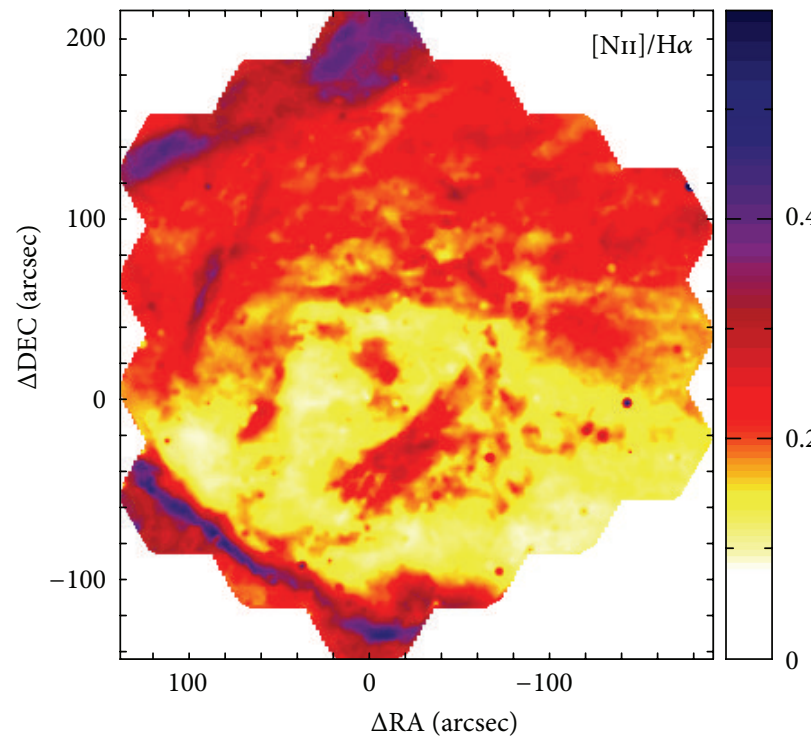

(c)

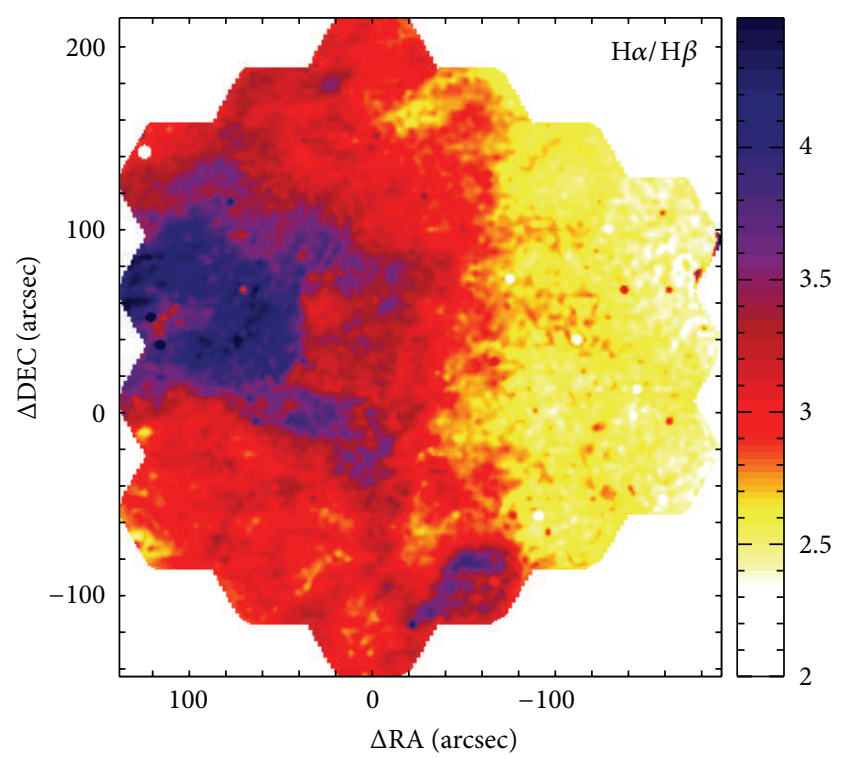

(b)

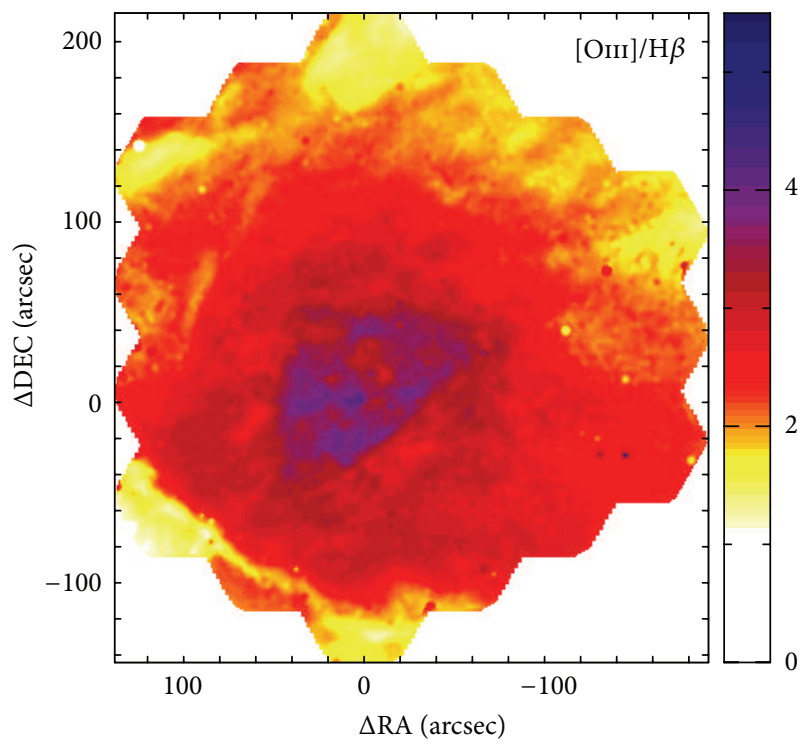

(d)

FIGURE 1: (a) Observed $\mathrm{H} \alpha$ Line intensity map in units of $10^{-12} \mathrm{erg} \mathrm{s}^{-1} \mathrm{scm}^{2} \operatorname{arcsec}^{-2}$ obtained by fitting a single Gaussian function to the $\mathrm{H} \alpha$ emission line for each single spectrum in the dataset. The intensity map is then reconstructed by interpolating the recovered flux at the location of each spectrum, as described in the text. (b) Observed $\mathrm{H} \alpha / \mathrm{H} \beta$ line ratio map. ((c) and (d)) Classical diagnostic line ratio maps. (c) $[\mathrm{NiI}] \lambda 6583 / \mathrm{H} \alpha$ line ratio map. (d) $[\mathrm{OIII}] \lambda 5007 / \mathrm{H} \beta$ line ratio map.

the abundance and ionizing condition can change across an Hir region, and, therefore, to be very precise, it is not correct to assign a particular abundance and defined ionizing conditions to the whole Nebula, as frequently done in study of extragalactic Hir regions. The comparison of the distribution of ionized conditions across the Orion Nebula with the ones derived for the integrated spectrum (coadding the $\sim 8000$ individual spectra) indicates that the integrated properties seem to be representative of the average ones across the Nebulae. However, it is still unclear how representative these average properties may be for different Hir regions.

\section{HII Regions and the Underlying Stellar Population}

Classical Hir regions are gas clouds ionized by short lived hot OB stars, associated with star formation. They are frequently selected on the basis of demarcation lines defined in the so-called diagnostic diagrams (e.g., $[18,22])$, which compare different line ratios, like $[\mathrm{OIII}] / \mathrm{H} \beta$ versus $[\mathrm{NII}] / \mathrm{H} \alpha$, $[\mathrm{OIII}] / \mathrm{H} \beta$ versus $[\mathrm{OII}] / \mathrm{H} \alpha,[\mathrm{NII}] / \mathrm{H} \alpha$ versus $[\mathrm{SII}] / \mathrm{H} \alpha$, and $/$ or $[\mathrm{NII}] / \mathrm{H} \alpha$ versus $[\mathrm{SII}] 6717,6731 / \mathrm{H} \alpha$. In most of the cases these ratios discriminate well between strong ionization sources, 
like classical Hir regions and powerful AGNs (e.g., [23]). However, they are less accurate in distinguishing between low-ionization sources, like weak AGNs, shocks, and/or postAGBs stars (e.g., $[24,25])$. Alternative methods based on a combination of the classical line ratios with additional information regarding the underlying stellar population have been proposed. For example, Cid Fernandes et al. [24] proposed the use of the $\mathrm{EW}(\mathrm{H} \alpha)$, to distinguish between retired (non-star-forming) galaxies, weak AGNs, and starforming galaxies.

Among the classical diagnostic diagrams, the most widely used is the diagram that uses the ratio between the strongest emission lines that are, at the same time, less affected by dust attenuation: $[\mathrm{OIII}] / \mathrm{H} \beta$ versus $[\mathrm{NII}] / \mathrm{H} \alpha$ [23]. We will refer hereafter to this diagnostic diagram as the BPT diagram along this study. Different demarcation lines have been proposed for this diagram. The most popular ones are the Kauffmann et al. [26] and Kewley et al. [27] curves. They are usually invoked to distinguish between star-forming regions (below the Kauffmann et al. [26] curve), and AGNs (above the Kewley et al. [27] curve) The location between both curves is normally assigned to a mixture of different sources of ionization. Additional demarcation lines have been proposed for the region above the Kewley et al. [27] curve to segregate between Seyfers and LINERs (e.g., [28]).

Despite the benefits of this clean segregation for classification purposes, it may introduce biases when trying to select HiI regions. The Kewley et al. [27] curve was derived on the basis of photoionization models. It corresponds to the maximum envelope in the considered plane for ionization produced by hot stars. Therefore, to the extent that these photoionization models are realistic, any combination of line ratios below this curve can be produced entirely by OB star photoionization. Finally it defines all the area above it as unreachable by ionization associated with photoionization by OB-stars directly associated with starformation. Other sources of ionization that may be related somehow with starformation at larger time scales, like shocks, ionization by Xray binaries are not contemplated in their photoionization models. However, the derived line ratios and/or morphological structure of this ionization are clearly different and in most cases above the considered demarcation line. The Kauffmann et al. [26] curve has a completely different origin. It is an empirical envelope defined to segregate between starforming galaxies and the so-called AGN branch in the BPT diagram based on the analysis of the emission lines for the SDSS galaxies. It describes well the envelope of classical HiI regions found in the disks of spiral galaxies. However, it is known that certain Hir regions can be found above this demarcation line as we will show below.

Kennicutt et al. [29] first recognized that Hir regions in the center of galaxies distinguish themselves spectroscopically from disk ones by their stronger low-ionization forbidden emission. The nature of this difference was not clear. It may be due to contamination by an extra source of ionization, like diffuse emission or the presence of an AGN. However, other stellar processes like nitrogen enhancement due to a natural aging process of HiI regions and the surrounding ISM can produce the same effect. These early results were confirmed by Ho et al. [30], who demonstrate that inner starforming regions may populate the right branch of the BPT diagram, at a location above the demarcation line defined latter by Kauffmann et al. [26]. However, we have found that Hir regions with similar ionisation characteristics are not restricted to the central regions and can be found at any galactocentric distances, even at more than 2 effective radii $\left(r_{e}\right)$, which excludes a possible contamination by the ionization of an AGN the origin of the detected line ratios. The nature of these HiI regions will be addressed in detail elsewhere. For the purpose of the current study it is important to define a selection criterion that does not exclude them. However, we would like to clarify that we do not find a correlation between the presence of these HII regions and other possible contaminating sources of ionization, like shocks and/or Active Galactic Nuclei (AGN).

Therefore, selecting HiI regions based on the Kauffmann et al. [26] curve may bias any sample towards classical disk regions, excluding an interesting population of these objects. On the other hand, it does not guarantee the exclusion of other sources of nonstellar ionizations that can populate this area, like shocks (e.g., [31, 32]), post-AGB stars (e.g., [25]), and dusty AGNs (e.g., [33]). Following Cid Fernandes et al. [34] and Cid Fernandes et al. [24], we consider that an alternative method to distinguish between different sources of ionization is to compare the properties of the ionized gas with those of the underlying stellar population. Cid Fernandes et al. [34] used the $\mathrm{EW}(\mathrm{H} \alpha)$ in combination with the $[\mathrm{NII}] / \mathrm{H} \alpha$ line ratio to distinguish between strong and weak ionization sources and between starformation and AGNs.

In Sanchez et al. ([35], submitted) we adopted a different selection criteria, using the fraction of young stars $\left(f_{y}\right)$ provided by the multi-Simple Stellar Population (SSP) analysis of the underlying stellar population, as a proxy of the starformation activity. For starforming regions this parameter provides a similar information as the $\mathrm{EW}(\mathrm{H} \alpha)$. Figure 2(a) shows the distribution of $\mathrm{EW}(\mathrm{H} \alpha)$ against the fraction of young stars for the $\sim 7000$ clumpy ionized regions selected by HIIEXPLORER. For those regions with $\operatorname{EW}(\mathrm{H} \alpha)>6 \AA$, and/or with a fraction of young stars larger than $20 \%$, both parameters present a strong log-linear correlation $\left(r_{\text {corr }}=\right.$ 0.95).

The threshold imposed by HIIExplorer on the surface brightness of $\mathrm{H} \alpha$ and the requirement that the distribution of emission is clumpy remove efficiently most of the ionization corresponding to weak-emission lines described. This is mostly diffuse emission that peaks in the described diagram at $\mathrm{EW}(\mathrm{H} \alpha) \sim 1-2 \AA$ and $f_{y} \sim 5-10 \%$. This weak $\mathrm{EW}(\mathrm{H} \alpha)$ is dominated mostly by post-AGB stars (e.g., $[25,36]$ ), and, therefore, no correlation is expected between its intensity and the fraction of young stars. On the other hand, high $\mathrm{EW}(\mathrm{H} \alpha)$ could be produced by other mechanisms, like AGNs and shocks, that do not require to be correlated with the properties of the underlying stellar population. A cut in the $\mathrm{EW}(\mathrm{H} \alpha)$ cannot remove those regions. Therefore, we consider that the fraction of young stars provides an indicator of recent star-formation activity.

Figure 3(a) shows the distribution of the ionized regions across the BPT diagram, indicating with contours the density 


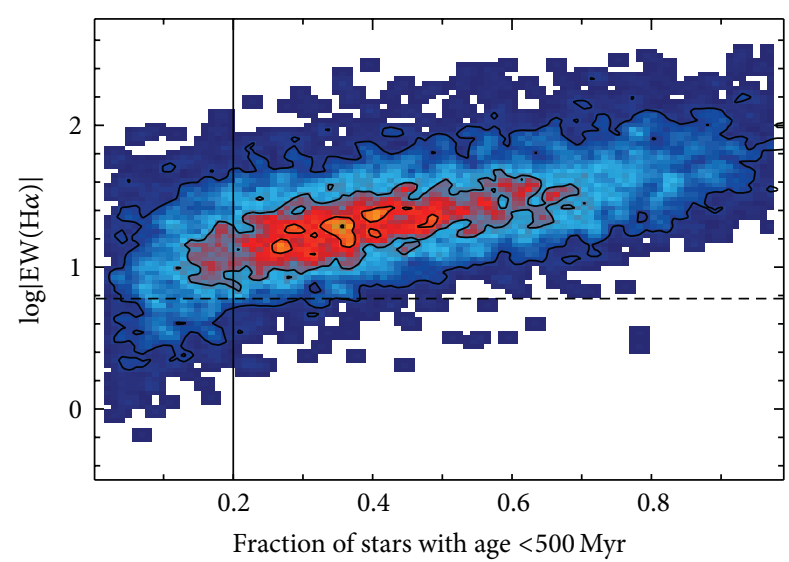

(a)

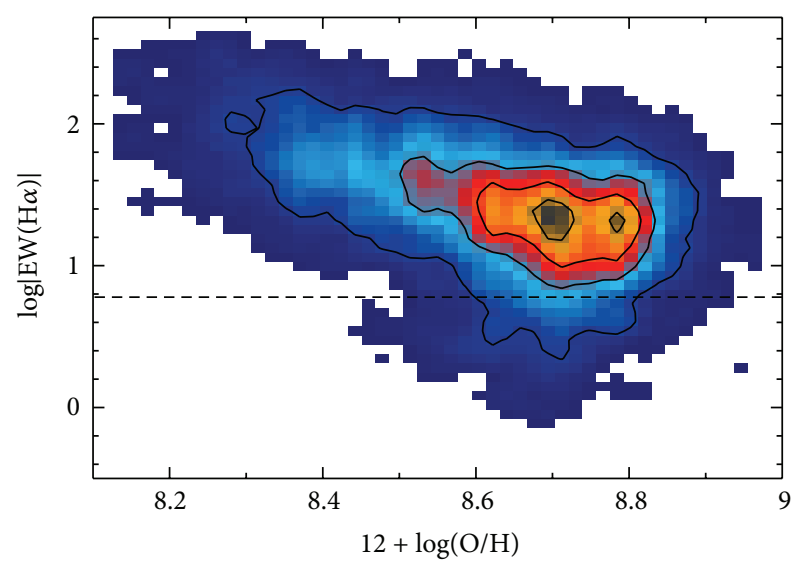

(b)

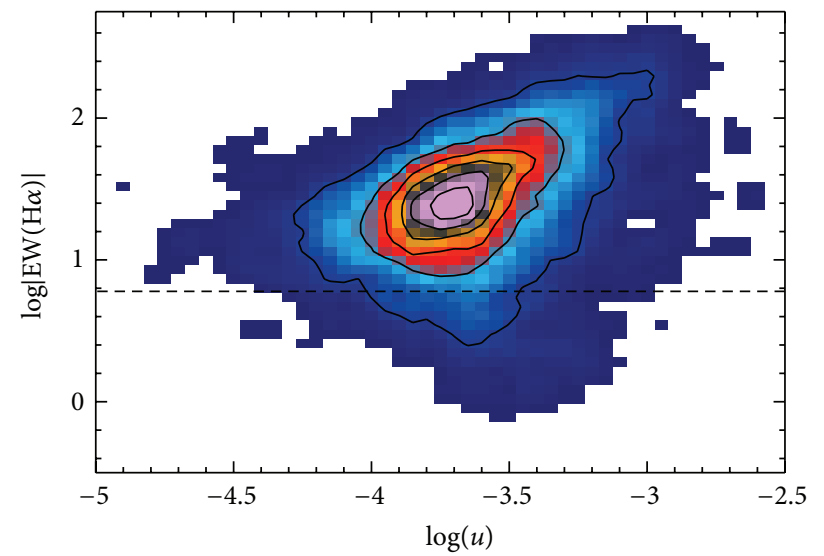

(c)

FIgURE 2: (a) Absolute value of the equivalent width of $\mathrm{H} \alpha$, in logarithmic scale, plotted against the luminosity fraction of young stars in the $V$-band, in the underlying stellar population (according to the SSP modelling by FIT3D) for the clumpy ionized regions selected with HIIExplorer. (b) Absolute value of the equivalent width of $\mathrm{H} \alpha$, in logarithmic scale, plotted against the oxygen abundance for the same ionized regions. (c) Absolute value of the equivalent width of $\mathrm{H} \alpha$, in logarithm scale, plotted against the ionization strength for the same ionized regions.

of regions at each location. The outermost of those contours encloses $95 \%$ of the detected regions, with each consecutive one encircling less regions. This contour is located below the Kewley et al. [27] demarcation curve, which indicates that the ionization of our selected clumpy regions is already dominated by star formation. Indeed, only 2\% of all regions are located above the Kewley et al. [27] line, and $\sim 80 \%$ are below the Kauffmann et al. [26] line (i.e., where classical disk Hir regions are located). If we had adopted this latter demarcation curve as our selection criteria, we would have missed a significant number of regions.

The color code in Figure 3 indicates the average luminosity fraction of young stars in the $V$-band at each location (i.e., the $x$-axis in Figure 2), ranging from nearly $100 \%$ for the regions at the top-left area of the diagram to nearly $0 \%$ for regions at the top-right location.

Based on these results, we classified as Hir regions those clumpy ionized regions for which young stars (<500 Myr) contribute at least a $20 \%$ to the flux in the $V$-band. This particular fraction is the lowest for which the correlation coefficient between $f_{y}$ and the $\operatorname{EW}(\mathrm{H} \alpha)$ is still higher than $r_{\text {corr }}>0.95$ and for which the fraction of excluded regions is not higher than the one that would be excluded by adopting the more common Kauffmann et al. [26] curve. We would like to stress that we are not considering that any star younger than 500 Myr may produce photoionization. The actual age of the ionizing population should be much younger (<20 Myr), in order to supply enough ionizing photons. However, the derived age of the underlying population does not depend only on the physical properties of that population but also on our ability to distinguish between different ages. This in turn depends on the $\mathrm{S} / \mathrm{N}$ of the continuum, its spectral resolution, the covered wavelength range, and the selected SSP-template. For many Hir regions, the continuum has a poor $\mathrm{S} / \mathrm{N}$, and therefore we prefer to adopt a very crude SSP library with just 4 ages (and 3 metallicities). Of them, a 50\% are younger than 500 Myrs and the other ones older than this age. Our spectral fit assigns all the blue component to ages younger than this limit, which, obviously, should not be interpreted as the true age of the ionizing population at all. 


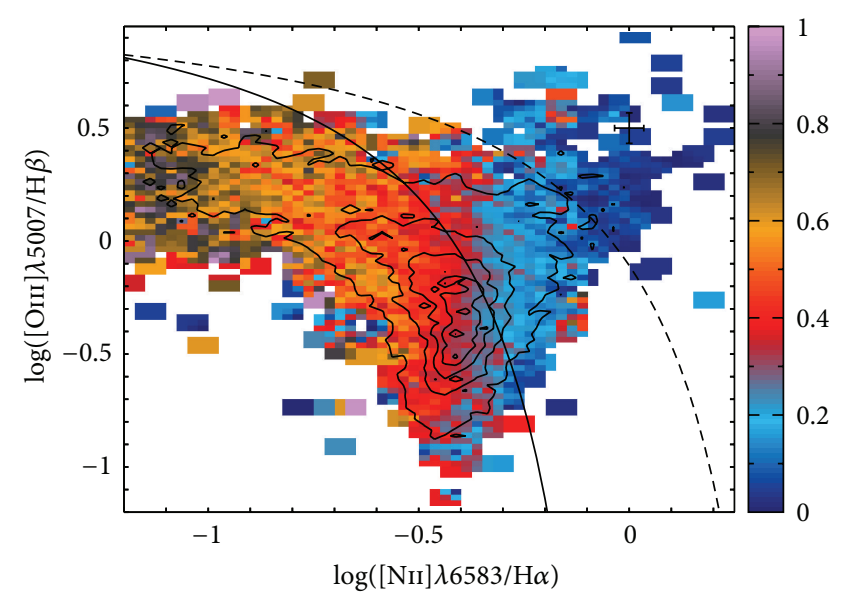

(a)

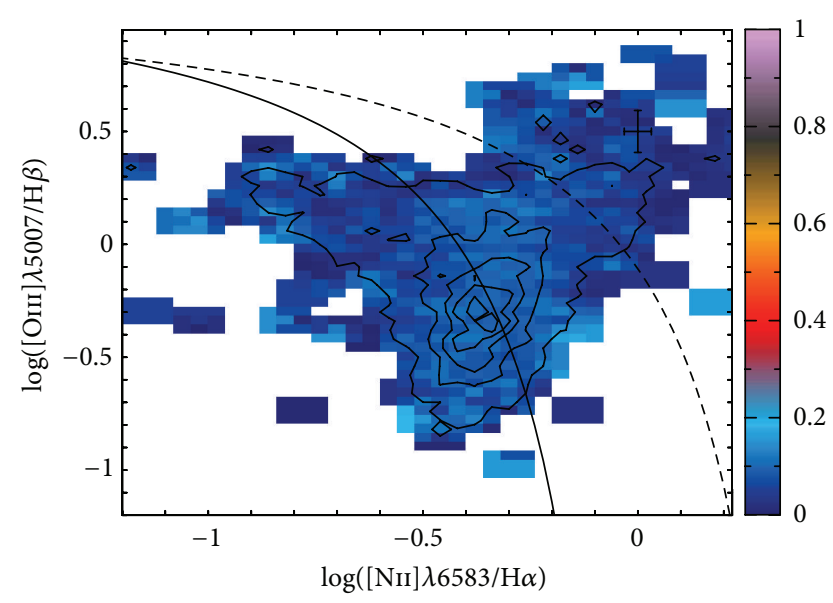

(b)

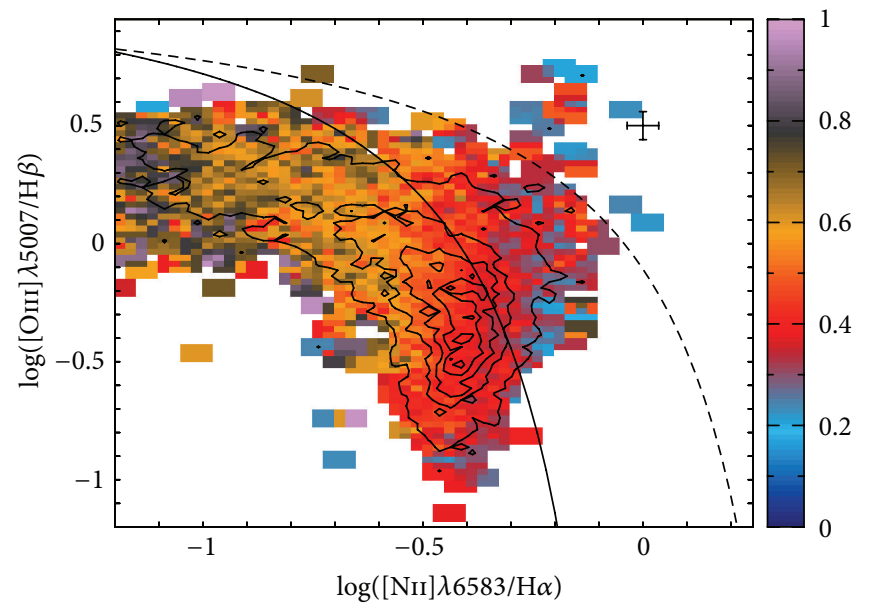

(c)

Figure 3: (a) [OIII] $\lambda 5007 / \mathrm{H} \beta$ versus [NII] $\lambda 6583 / \mathrm{H} \alpha$ diagnostic diagram for the $\sim 7000$ ionized regions described in the text. The contours show the density distribution of these regions with the diagram plane, with the outermost contour enclosing $95 \%$ of the regions, and each consecutive one enclosing $20 \%$ less regions. The color indicates the fraction of young stellar population in the underlying continuum. (b) The same diagnostic diagram restricted to those ionized regions with less than a $20 \%$ of young stellar population ( 1800 regions). (c) The same diagnostic diagram restricted to those ionized regions with more than a $20 \%$ of young stellar population ( $\sim 5800$ regions). In all the panels, the solid- and dashed-line represent, respectively, the Kauffmann et al. [26] and Kewley et al. [27] demarcation curves. They are usually invoked to distinguish between classical star-forming objects (below the solid line) and AGN powered sources (above the dashed line). Regions between both lines are considered intermediate ones.

Figure 3(b) shows the same distribution as the one shown in Figure 3(a) but restricted to the 1787 regions for which the fraction of young stars is lower than $20 \%$. The fraction of regions above the Kewley et al. [27] curve is significantly larger $(\sim 7 \%)$, with more than a $40 \%$ above the Kauffmann et al. [26] one. Although there are still 1043 regions below this latter curve, they comprise just $\sim 15 \%$ of the original sample. We consider this fraction as the incompleteness limit of detected Hir regions.

Figure 3(c) shows the same distribution, but for the 5229 regions with a fraction of young stars larger than $20 \%$, that is, our final sample of Hir regions. Of them, only 23 are above the Kewley et al. [27] curve ( $\sim 99.5 \%$ are below it). On the other hand, there are 713 regions in the so-called intermediate region, with a significant fraction of young stars $(\sim 40 \%$ on average). These regions would have been excluded if we had adopted the Kauffmann et al. [26] curve as our selection criteria, losing Hir at any galactocentric distance.

Figures 3 and 2(a) indicate that the location of an HII region in the BPT diagram is strongly correlated with the properties of the underlying stellar population. It is important to recall here that the $\operatorname{EW}(\mathrm{H} \alpha)$ is a proxy of the specific star-formation rate (sSFR) or the current star-formation compared to the total stellar mass (e.g., [37]). Evenmore, the fraction of young stars is a direct measurement of the most recent star-forming activity. Therefore, the $\mathrm{EW}(\mathrm{H} \alpha)$ and $f_{y}$ are tracers of the composition of the underlying stellar population and the recent star-formation history of the galaxy at the location of the HII region. We must mention also that both parameters are measured including the line-of-sight underlying stellar population, since the extraction of the spectra is performed on simple apertures 
(segmented regions) and therefore these spectra comprise both young and old stars (although only the young ones are responsible of the gas ionization).

Under the assumption of a particular IMF, a particular burst (instantaneous or continuous), a particular dust model, and a fixed evolutionary track for the stars and the prescriptions for the stellar atmosphere (which are model dependent), the location in the BPT diagram is well defined by two parameters: (i) the ionization strength, $u$ (directly related to the ionization parameter), and (ii) the metallicity (e.g., [27]). This is clearly illustrated in Figure 3, where the distribution of the same ionized regions is shown as in Figure 3, without discriminating between different sources of ionization, together with the expected location for two ionization models produced with MAPPINGS-III [27, 38], under certain reasonable assumptions for the dust attenuation and adopting the evolutionary tracks for the ionizing population derived from the PEGASE code [39]. The models assume a fixed electron density of $n_{e}=350 \mathrm{~cm}^{-3}$ (near the average value reported for our Hir regions) and a set of ionization strengths and metallicities. Two model grids are considered, one assuming an instant starburst at a zero age (left panel) and the other assume a continuous starformation process, which is represented at $4 \mathrm{Myr}$ (right panel). The ionized regions located within the BPT diagram in the area described by the classical His regions in the disk of galaxies are characterized well by models comprising a single instataneus starburst, with different ionization strengths and metallicities. However, those ionized regions located above the Kauffmann et al. [26] demarcation line can be reproduced only if a continuous starformation is assumed. A similar behavior was reported by Terlevich et al. [40], when analyzing the age of Hir galaxies, that corresponds much better with a sequence of star-formation events rather than with a single burst. It seems that the Hir regions located in the inner-most areas and in the older galaxies present a similar behavior.

In any case, it is clear that the location in the BPT diagram is directly related to the ionization strength and metallicity. On the other hand, in Figure 3 this location is directly related with the properties of the underlying stellar population, weighted by the $\mathrm{EW}(\mathrm{H} \alpha)$ or the fraction of young stars, parameters that are strongly correlated (Figure 2). Taking both results together, a strong correlation is expected between the properties of the underlying stellar population and both the ionization strength and the gas abundance. Indeed, this is the case. Figures 2(a) and 2(b), shows the clear correlation between the $\operatorname{EW}(\mathrm{H} \alpha)$ and both the gas abundance and the ionization strength, directly derived from different emission line ratios (see [14], for details on the calculations). Similar correlations are found with the fraction of young stars, $f_{y}$, defined before. Thus, although His regions are short-lived events, its ionization conditions appear to be strongly related to the preexisting underlying stellar population and therefore to the overall star-formation history at their location.

\section{Highlights on Our Previous Results}

Our program to understand the star-formation processes and chemical evolution of galaxies based on the study of ionized gas in Hir regions has two approaches. In the first one we focused on the study of the ionization processes within individual Hir regions in our Galaxy, like Orion Nebula [15] or M1-67 [16]. In the second one we focused on the study of the statistical properties of large samples of extragalactic HII regions, the main highlights of which we summarize here.

This statistical program was initiated with the PINGS survey [12], which acquired IFS mosaic data for a number of medium size nearby galaxies. In Sánchez et al. [41] and Rosales-Ortega et al. [42] we studied in detail the ionized gas and Hir regions of the largest galaxy in the sample (NGC 628). The main results of these studies are included in the contribution by Rosales-Ortega in the current edition. We then continued the acquisition of IFS data for a larger sample of visually classified face-on spiral galaxies [43], as part of the feasibility studies for the CALIFA survey [13]. The spatially resolved properties of a typical galaxy in this sample, UGC9837, were presented by [44].

In Sánchez et al. [14] we presented a new method to detect, segregate, and extract the main spectroscopic properties of HiI regions from IFS data (HIIEXPLORER (http://www .caha.es/sanchez/HII_explorer/)). In this paper a catalog of 2600 Hir regions and aggregations extracted from 38 faceon spiral galaxies compiled from the PINGS and CALIFA feasibility studies was presented. Using this catalog we found a new local scaling relation between the stellar mass density and oxygen abundance, the so-called $\Sigma-Z$ relation, that can naturally explain the well-known mass-metallicity relation [45]. This relation is consistent with an inside-out growth scenario for disk-dominated galaxies, supported by different observational results based on photometric studies (e.g., [4649]), classical slit spectroscopy (e.g., $[50,51])$, and recent IFS results (e.g., $[52,53])$.

The same catalog allows us to explore the galactocentric radial gradient of the oxygen abundance [14]. Models based on the standard inside-out scenario of disk formation predict a relatively quick self-enrichment with oxygen abundances and an almost universal negative metallicity gradient once this is normalized to the galaxy optical size Boissier and Prantzos [54, 55]. In Sánchez et al. [14], we confirmed that up to $\sim 2$ disk effective radii there is a negative gradient of the oxygen abundance in all the analyzed spiral galaxies. The gradient presents a very similar slope for all the galaxies $\left(\sim-0.12 \mathrm{dex} / r_{e}\right)$, when the radial distances are measured in units of the disk effective radii. We found no difference in the slope for galaxies of different morphological types: early/late spirals, barred/nonbarred, and grand-design/flocculent.

Beyond $\sim 2$ disk effective radii our data show evidence of a flattening in the abundance, consistent with several other spectroscopic explorations, based mostly on single objects (e.g., [42, 56-59]). The same pattern in the abundance has been described in the case of the extended UV disks discovered by GALEX $[60,61]$, which show oxygen abundances that are rarely below one-tenth of the solar value. Additional results, based on the metallicity gradient of the outer disk of NGC 300 from single-star CMD analysis [62], or spectroscopic analysis of the HiI region NGC 2579 in the outer regions of the Milky-Way [63], support the presence of a flatter gradient towards the outer disk. Despite all these 


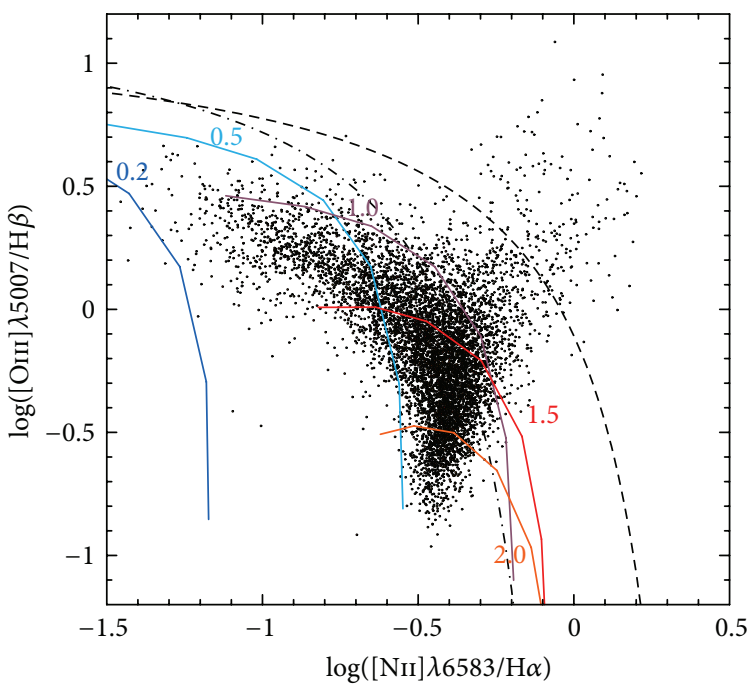

(a)

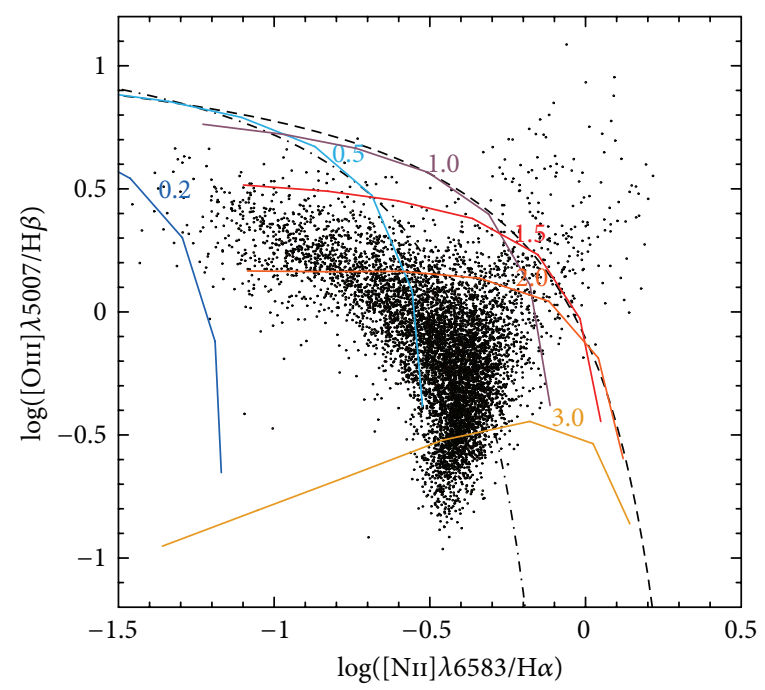

(b)

Figure 4: (a) [OIII] $\lambda 5007 / \mathrm{H} \beta$ versus [NiI] $\lambda 6583 / \mathrm{H} \alpha$ diagnostic diagram for the $\sim 7000$ ionized regions shown in Figure 3(a). The color solidlines represent the expected line ratios derived from the MAPPINGS-III ionization models, based on an instantaneous zero-age starbust model based on the PEGASE spectral energy distribution. Each solid-line corresponds to a different metallicity, which is indicated with the corresponding label in units of solar abundance, with a different ionization strength that varies from strong to weak ionizations from left to right in each line. (b) Identical diagram to that in (a), with the only difference that in this particular case the color solid-lines represents the expected line ratios for a continuous starburst model after $4 \mathrm{Myr}$ of its ignition. In ((a), (b)), the dot-dashed and dashed-line represent, respectively, the Kauffmann et al. [26] and Kewley et al. [27] demarcation curves. They are usually invoked to distinguish between classical star-forming objects (below the solid line) and AGN powered sources (above the dashed line). Regions between both lines are considered intermediate ones.

results, the outermost parts of the disk have not been explored properly, either due to the limited number of objects explored by the previous studies or due to the limited spatial coverage (e.g., [14]).

To further explore these issues, we applied the same procedure to the IFS data provided by the CALIFA survey [13] (http://califa.caha.es/). CALIFA is an ongoing exploration of the spatially resolved spectroscopic properties of galaxies in the Local Universe $(z<0.03)$ using wide-field IFS to cover the full optical extent (up to $\sim 3-4 r_{e}$ ) of $\sim 600$ galaxies of any morphological type, distributed across the entire color-magnitude diagram (Walcher et al., in prep.), and sampling the wavelength range 3650-7500 $\AA$. So far, the survey has completed $\sim 1 / 2$ of its observations, with 306 galaxies observed (May 2013), and the first data release, comprising 100 galaxies, was already delivered in November 2012 by Husemann et al. [64].

In Sánchez et al. [35] we presented the first results based on the catalog of HiI regions extracted from these galaxies. In Here, we studied the dependence of the $\mathscr{M}-Z$ relation with the star-formation rate, for the 113 galaxies with enough HII regions to derive the characteristic oxygen abundance. We found no secondary relation different than the one induced by the well-known relation between the star formation and the mass, contrary to what was claimed by other authors $[65,66]$, based on single aperture spectroscopic data (SDSS). Although the reason for the discrepancy is still not clear, we postulate that simple aperture bias, like the one present in previous datasets, may induce the reported secondary relation.
Figure 6 presents an updated version of these results, including the last list of analyzed galaxies (193 galaxies from the CALIFA sample together with 31 galaxies from the CALIFApilot studies). Figure 4 (a) shows the $\mathscr{M}-Z$ relation found for these galaxies, with color code indicating the integrated SFR for each galaxy. It is appreciated that the stronger gradient in SFR be along the stellar mass, as expected for star-forming galaxies. Once the best fitted function to the $\mathscr{M}-Z$ relation subtracted, the residual of the abundance does not present any evident secondary relation with the SFR (as it is seen in Figure 4(b)). That is, the results presented in [35] are confirmed with a sample of galaxies enlarged by almost a factor two.

We also confirmed the local $\sum$ - $Z$ relation unveiled by [42], with a larger statistical sample of HiI regions ( 5000). Both the $\Sigma-Z$ relation, the evidence for a common slope in the gradient of the oxygen abundance, and the lack of dependence of the $\mathscr{U}-Z$ relation with the star-formation rate are consistent with chemical evolution dominated by an insideout growth with little/limited influence of processes that redistribute metals, like out flows and/or radial migrations.

In Sanchez et al. [67] we used the updated CALIFA catalog of $\sim 5000$ HiI regions, extracted from 193 individual galaxies shown in Figure 6, to study the radial oxygen abundance gradient up to 3-4 disk effective radii. We confirmed that the abundance gradients present a common slope up to $\sim 2$ effective radii, with a distribution compatible with that being produced by random fluctuations, for all galaxies when normalized to the disk effective radius of $\alpha_{\mathrm{O} / \mathrm{H}}=-0.1 \mathrm{dex} / r_{e}$. 

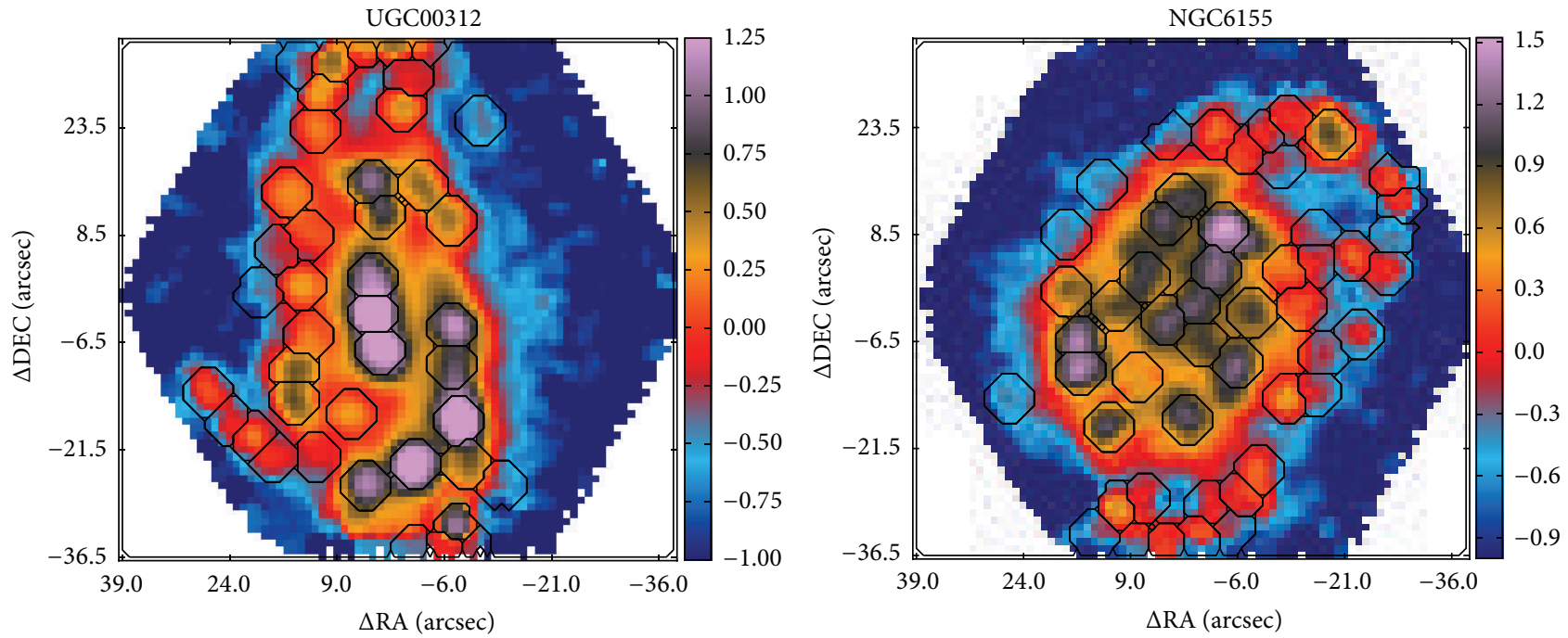

(a)
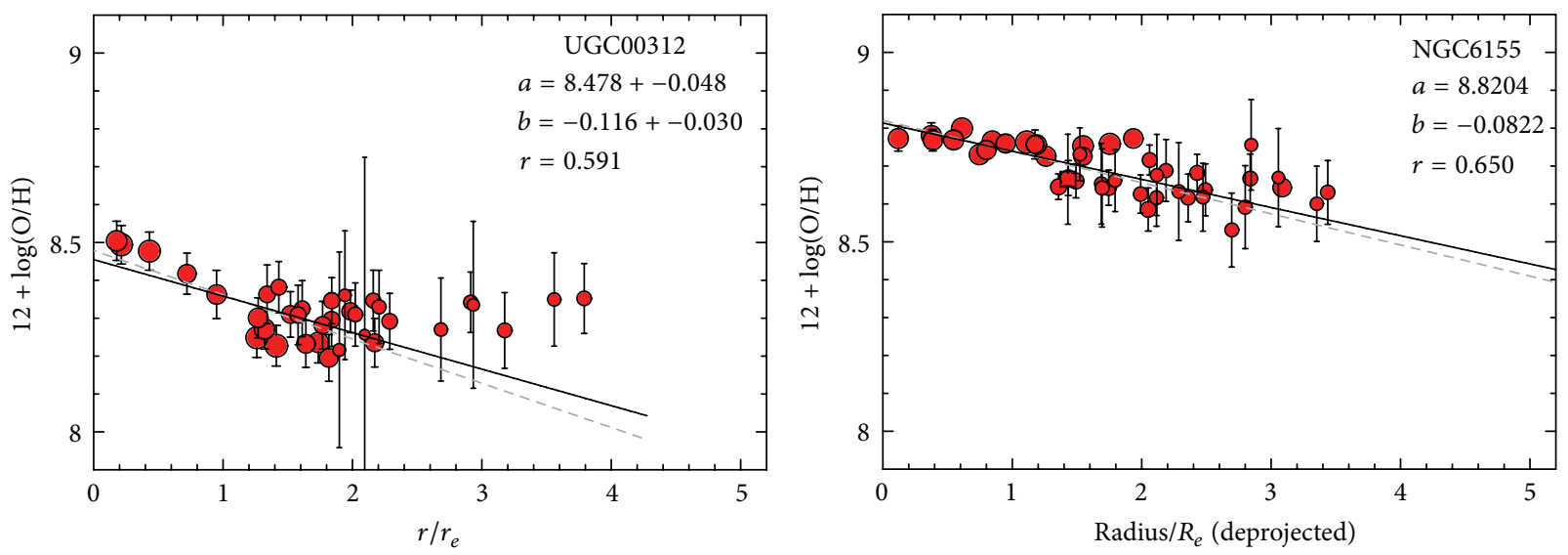

(b)

FIGURE 5: (a) IFS-based $\mathrm{H} \alpha$ maps, in units of $10^{-16} \mathrm{erg} \mathrm{s}^{-1} \mathrm{~cm}^{-2} \operatorname{arcsec}^{-2}$, derived for two representative galaxies of the sample (color images), together with the detected HiI regions shown as black segmented contours. (b) Radial distribution of the oxygen abundance derived for the individual Hir regions with abundance errors below $0.15 \mathrm{dex}$, as a function of the deprojected distance (i.e., corrected for inclination), normalized to the effective radius, for the same galaxies. The size of the circles is proportional to the $\mathrm{H} \alpha$ intensity.

Figure 5 shows the segregation map based on $\mathrm{H} \alpha$ intensity to define the Hir regions, and the radial gradient in the oxygen abundance two galaxies in our sample (a poor metal and a rich one). No significant differences are found on the basis of the morphological type, presence or absence of bars, absolute magnitude and/or stellar mass. The only clear deviation from the common slope is seen in galaxies with evidence of interaction or undergoing a merging process. For these galaxies the gradient is significantly flattered. change. These results agree with the main conclusion of our previous study [14], where a limited sample of 38 face-on spiral galaxies was analysed, using similar methods as the ones described here. In a companion article (Sánchez-Blazquez [51] in prep.), we analyse the radial gradient of stellar metallicity, where we have found consistent results.

Beyond $\sim 2 r_{e}$ our data reveal clear evidence of a flattening in the abundance in most of the galaxies with detected HII regions at this radial distances. A change in the slope of the radial gradient of oxygen abundance has been reported by several authors (e.g., $[14,56,58,59]$ ), although with less significant numbers. We cannot provide a conclusive answer regarding the origin of this flattening.

\section{Evidence for an Inside-Out Growth}

Along this paper we described the different results we have obtained in our studies of HiI regions using IFS. Our main results can be summarized as follows.

(i) HII regions present a large variety of properties across their optical extent, both with regard to the ionization strength and gas abundances, and they do not seem to be fully ionized everywhere across their entire optical extent (as seen in the IFU data of the Orion Nebula). 


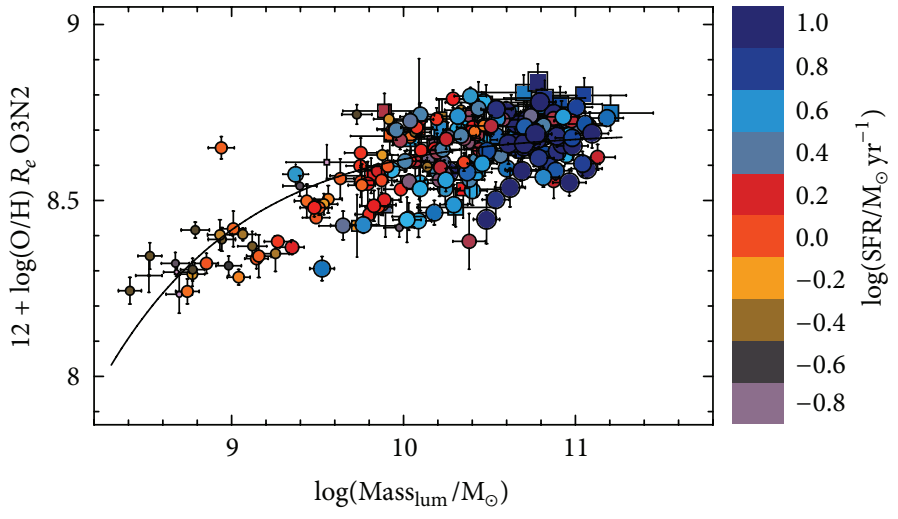

(a)

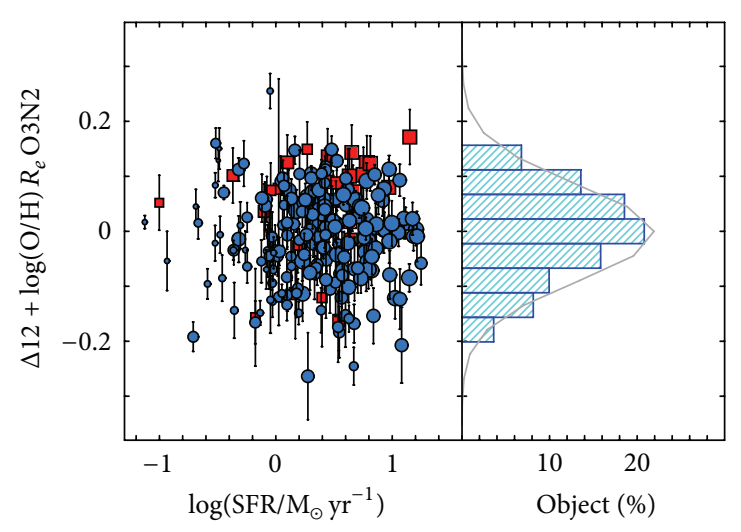

(b)

FIgURE 6: (a) Distribution of the oxygen abundances at the effective radii as a function of the integrated stellar masses for the CALIFA galaxies (193, circles). For comparison purposes, we show similar values for the galaxies observed in the CALIFA feasibility studies (31, squares) included in the HiI regions catalog described in Sánchez et al. [14]. The size of the symbols is proportional to the integrated SFR. The solid line represents the best-fitting curve, as described in Sanchez et al. [35]. The colors represent the logarithm of the integrated SFR for each galaxy. (b) Distribution of the differential oxygen abundances with respect to the solid-line shown in (a) (i.e., the dependence on the stellar mass), as a function of the integrated SFR for the CALIFA galaxies (113, blue solid circles). The size of the symbols is proportional to the oxygen abundances shown in Figure 6. The histogram shows the same distribution of differential oxygen abundances. The solid line represents a Gaussian function with the same central value $(0.01 \mathrm{dex})$ and standard deviation $(0.07 \mathrm{dex})$ as the represented distribution, scaled to match the histogram.

(ii) The spectroscopic properties derived using integrated spectra over large areas of Hir regions seem to be representative of the average properties derived when analysing individual spectra across their optical extent.

(iii) The ionization strength and gas abundance and therefore the location of the HiI regions in the different diagnostic diagrams correlate with the properties of their underlying stellar population and thus reflect the local star-formation history.

(iv) The gas abundance presents a negative radial gradient, with more metal rich Hir regions in the inner areas and more metal poor ones in the outer ones. Independently of the galaxy properties all galaxies present a common slope for this gradient up to 2 effective radii of $\alpha_{\mathrm{O} / \mathrm{H}}=-0.1 \mathrm{dex} / r_{e}$, when the galactocentric distance is normalized to the effective radius of the disk.

(v) The gas abundance is strongly correlated with the stellar surface mass density ( $\sum-Z$ relation), following a correlation similar to the $\mathscr{U}-Z$ relation.

(vi) Our high-quality data do not reveal any evidence for a secondary relation between the $\mathscr{M}-Z$ relation (or the $\sum-Z$ relation) with the star-formation rate (or the SFR density), different than the well-known relation between the SFR and the stellar mass for star-forming galaxies.

The existence of a universal radial decrease in the oxygen abundance has been already reported in many previous studies (e.g., $[5,9,14,42,56-59])$. This observational property is compatible with our current understanding of the formation and evolution of spiral galaxies (e.g., [68] and the references therein). Gas accretion brings gas into the galactic center, where it first reaches the required density to ignite star formation. Thus the inner regions are populated by older stars, and they have undergone a faster gas reprocessing, and galaxies experience an inside-out mass growth (e.g., $[54,69])$. Both the extinction-corrected color gradients in nearby galaxies, [49] and weak dependence of the masssize relation with redshift [46-48] support an inside-out scenario for the evolution of disks. Recent results, based the on analysis of the star-formation history of CALIFA data, found undisputed evidence of the inside-out growth of the stellar mass in galaxies [52], at least for galaxies more massive than $7 \times 10^{10} \mathrm{M}_{\odot}$. These results are also supported by the radial distribution of the stellar ages found for the same dataset [53].

However, the described characteristic slope for the abundance gradient, independent of many of the properties of the galaxies, was only recently proposed [14]. This result imposes a more severe restriction to our current understanding of how disk galaxies growth. In essence, it agrees with the recently proposed $\Sigma-Z$ relation [45] that links the gas abundance with the mass density of the underlying stellar population. It describes how the stellar mass and the gas abundance, both fundamental products of the star formation history, growth consistently in disk galaxies, from the center to the outer-parts. Together with the $\mathscr{M}-Z$ relation, they indicate that more massive galaxies (that trace the strongest potential wells) are formed before and faster, accumulating more stellar mass and more metals. The presence of a common gradient in the abundance indicates that all disk dominated galaxies of the same disk effective radius (hence, the same disk mass) build up their metal content at a given normalized radius with a similar efficiency. 
The common slope suggests that the chemical evolution of galaxies is very similar in all disk galaxies, being compatible with a closed-box model. The classical closed-box model considers that each radial bin of a galaxy comprises primordial gas from the which stars which are born, live all their life time, and die in-situ, according to a given SFR and IMF prescriptions [70]. Therefore, if the amount of primordial gas is proportional to the depth of the potential well and the efficiency of the SFR is the same for all the galaxies, both the stellar mass and the enrichment would be just proportional to the time, for a given halo mass. The lack of dependence of the $\mathscr{U}-Z$ and $\Sigma-Z$ relations with the SFR (or the SFR surface density) supports also this scenario. Under this assumption all galaxies should have a universal gradient of their oxygen abundance with its zero point being proportional to the total mass.

However, it is well known that the closed-box model cannot predict the right fraction of metal-poor stars with respect to the observed metallicity distribution of nearby long-lived stars in the Milky Way (e.g., [71]). A more realistic model overcomes this problem by allowing the disk of galaxies to form via continuous accretion gas, driven by the gravitational force. This accretion can be compensated or even overcome for certain galaxies and certain periods by supernova explosions (e.g., [72]). However, the outflow of gas is not expected to feature in the history of most spiral galaxies and is usually neglected in the models [71]. This modified model is consistent with the described common radial gradient if the local gas recycling is faster than other timescales involved [73], and if the radial inflow is similar for those radial bins with the same stellar mass.

Despite these evidences, other authors consider that galactic winds play a fundamental role in the shaping of spiral galaxies (e.g., [74]) and may be an essential ingredient in their formation. Galactic winds are normally associated with strong star-formation events, but there is also evidence for such winds at more moderate SFR. They seem to be a ubiquitous event in high redshift galaxies (e.g., $[75,76]$ ), and some theoretical work emphasizes that they are a fundamental ingredient in the evolution of galaxies (e.g., [77, 78]). In particular, they may play a role in the enrichment of the intergalactic medium, although in most of the cases they do not seem to escape the dark matter halo around the considered galaxy. However, our results indicate that gas flows seem to have a less relevant effect in the shape of the chemical gradient in galaxies than anticipated, that is, their net effect is not clearly evident.

Our results do not rule out the existence of radial gas motions and metal mixing, and they, however, add new constraints on their net effect on the chemical abundance patterns of disk galaxies. Contrary to the isolated disk galaxies, interacting/merging galaxies show a clearly flatter metallicity distribution, in agreement with Kewley et al. [79] and Rich et al. [80]. This indicates that galaxy interactions can result in an effective mixing of metals. The absence of a clear difference in the radial abundance slopes of barred and unbarred galaxies suggests that bars (i) do not significantly enhance the efficiency of metal mixing in galaxies or (ii) produce a roughly proportional change in the gas abundance and stellar mass distribution that compensates each other when normalized to the disk effective radius, or (iii) are of temporary nature and their lifetime is shorter than the timescale for chemical abundance mixing in galaxies.

In general, the properties of the ionized gas in late-type galaxies are consistent with a quiescent evolution, where gas recycling is faster than other times scales involved [73]. This would imply that the galaxies seem to behave more locally in a similar manner than globally dominated by a radial mass distribution following the potential well of the matter, with an inside-out growth that is regulated by gas inflow and local downsizing star formation. Therefore, the dominant parameter that defines the amount of metals is the stellar mass, since both parameters are the consequence of a (almost) closed-box star-formation process. However, we should remark that our results are only valid for galaxies with stellar masses higher than $\sim 10^{9.5} \mathrm{M}_{\odot}$, where the CALIFA sample becomes complete.

\section{Acknowledgments}

Sebastian F. Sánchez thanks the director of CEFCA, M. Moles, for his sincere support of this project. This study makes use of the data provided by the Calar Alto Legacy Integral Field Area (CALIFA) survey (http://califa.caha.es/). CALIFA is the first legacy survey being performed at Calar Alto. The CALIFA collaboration would like to thank the IAA-CSIC and MPIA-MPG as major partners of the observatory and CAHA itself, for the unique access to telescope time and support in manpower and infrastructures. The CALIFA collaboration also thanks the CAHA staff for the dedication to this project. Based on observations collected at the Centro Astronómico Hispano Alemán (CAHA) at Calar Alto, operated jointly by the Max-Planck-Institut für Astronomie and the Instituto de Astrof sica de Andalucia (CSIC). The author thanks the anonymous referee for the many useful and detailed comments. The author also thanks P. Papaderos for the useful comments and the suggestions to clean and clarify the text. We thank the Viabilidad, Diseño, Acceso y Mejora funding program, ICTS-2009-10, for supporting the initial development of this project. He thanks the Plan Nacional de Investigación y Desarrollo funding programs, AYA2010-22111C03-03 and AYA2010-10904E, of the Spanish Ministerio de Ciencia e Innovación, for the support given to this project. He thanks the the Ramón y Cajal project RyC-2011-07590 of the spanish Ministerio de Econom a y Competitividad, for the support give to this project. He acknowledges suuport by the Grants nos. M100031241 and M100031201 of the Academy of Sciences of the Czech Republic (ASCR internal support program of international cooperation projects-PIPPMS) and by the Czech Republic program for the long-term development of the research institution no. RVO67985815.

\section{References}

[1] Á. R. López-Sánchez, "Massive star formation in Wolf-Rayet galaxies: V. Star-formation rates, masses and the importance of galaxy interactions," Astronomy \& Astrophysics, vol. 521, article A63, 31 pages, 2010. 
[2] Á. R. López-Sánchez and C. Esteban, "Massive star formation in Wolf-Rayet galaxies: IV. Colours, chemical-composition analysis and metallicity-luminosity relations," Astronomy \& Astrophysics, vol. 517, article A85, 28 pages, 2010.

[3] J. Lequeux, M. Peimbert, J. F. Rayo, A. Serrano, and S. TorresPeimbert, "Chemical composition and evolution of irregular and blue compact galaxies," Astronomy \& Astrophysics, vol. 80, pp. 155-166, 1979.

[4] E. D. Skillman, "Empirical oxygen abundances and physical conditions for relatively low abundance Hir regions," Astrophysical Journal, vol. 347, pp. 883-893, 1989.

[5] M. B. Vila-Costas and M. G. Edmunds, "The relation between abundance gradients and the physical properties of spiral galaxies," Monthly Notices of the Royal Astronomical Society, vol. 259, no. 1, pp. 121-145, 1992.

[6] D. Zaritsky, R. C. Kennicutt Jr., and J. P. Huchra, "Hiı regions and the abundance properties of spiral galaxies," Astrophysical Journal Letters, vol. 420, no. 1, pp. 87-109, 1994.

[7] C. A. Tremonti, T. M. Heckman, G. Kauffmann et al., "The origin of the mass-metallicity relation: insights from 53,000 starforming galaxies in the sloan digital sky survey," Astrophysical Journal Letters, vol. 613, no. 2, pp. 898-913, 2004.

[8] D. R. Garnett, "The luminosity-metallicity relation, effective yields, and metal loss in spiral and irregular galaxies," Astrophysical Journal Letters, vol. 581, no. 2, pp. 1019-1031, 2002.

[9] A. I. Diaz, "Abundance gradients in disc galaxies and chemical evolution models," in Evolutionary Phenomena in Galaxies, J. E. Beckman and B. E. J. Pagel, Eds., pp. 377-397, 1989.

[10] P. Martin and J.-R. Roy, "The influence of bars on the chemical composition of spiral galaxies," Astrophysical Journal Letters, vol. 424, no. 2, pp. 599-614, 1994.

[11] J. Moustakas and R. C. Kennicutt Jr., "An integrated spectrophotometric survey of nearby star-forming galaxies," Astrophysical Journal Supplement Series, vol. 164, no. 1, pp. 81-98, 2006.

[12] F. F. Rosales-Ortega, R. C. Kennicutt Jr., S. F. Sánchez et al., "PINGS: the PPAK IFS nearby galaxies survey," Monthly Notices of the Royal Astronomical Society, vol. 405, pp. 735-758, 2010.

[13] S. F. Sánchez, R. C. Kennicutt Jr., A. Gil de Paz et al., "CALIFA, the Calar Alto Legacy Integral Field Area survey: I. Survey presentation," Astronomy \& Astrophysics, vol. 538, article A8, 2012.

[14] S. F. Sánchez, F. F. Rosales-Ortega, R. A. Marino et al., "Integral field spectroscopy of a sample of nearby galaxies: II. Properties of the H II regions," Astronomy \& Astrophysics, vol. 546, article A2, 28 pages, 2012.

[15] S. F. Sánchez, N. Cardiel, M. A. W. Verheijen et al., "PPAK integral field spectroscopy survey of the Orion nebula," Astronomy \& Astrophysics, vol. 465, no. 1, pp. 207-217, 2007.

[16] A. Fernández-Martín, J. M. Vílchez, E. Pérez-Montero et al., "Integral field spectroscopy of M1-67. A Wolf-Rayet nebula with luminous blue variable nebula appearance," Astronomy \& Astrophysics, vol. 554, article A104, 15 pages, 2013.

[17] J. B. Kaler, "A catalog of relative emission line intensities observed in planetary and diffuse nebulae," Astrophysical Journal Supplement Series, vol. 31, no. 688, p. 517, 1976.

[18] J. A. Baldwin, G. J. Ferland, P. G. Martin et al., "Physical conditions in the orion nebula and an assessment of its helium abundance," Astrophysical Journal Letters, vol. 374, no. 2, pp. 580-609, 1991.

[19] D. E. Osterbrock, Astrophysics of Gaseous Nebulae and Active Galactic Nuclei, University Science Books, Mill Valley, Calif,
USA, 1989, Research supported by the University of California, John Simon Guggenheim Memorial Foundation, University of Minnesota, et al.

[20] B. E. J. Pagel, M. G. Edmunds, D. E. Blackwell, M. S. Chun, and G. Smith, "On the composition of H II regions in southern galaxies. I-NGC 300 and 1365," Monthly Notices of the Royal Astronomical Society, vol. 189, pp. 95-113, 1979.

[21] R. W. Pogge, J. M. Owen, and B. Atwood, "Imaging spectrophotometry of the Orion Nebula core. I-emission-line mapping and physical conditions," Astrophysical Journal, vol. 399, no. 1, pp. 147-158, 1992, Erratum in "imaging spectrophotometry of the Orion Nebula core. I-emission-line mapping and physical conditions", Astrophysical Journal, vol. 408, p. 758, 1993.

[22] S. Veilleux and D. E. Osterbrock, "Spectral classification of emission-line galaxies," Astrophysical Journal Supplement Series, vol. 63, pp. 295-310, 1987.

[23] J. A. Baldwin, M. M. Phillips, and R. Terlevich, "Classification parameters for the emission-line spectra of extragalactic objects," Publications of the Astronomical Society of the Pacific, vol. 93, pp. 5-19, 1981.

[24] R. Cid Fernandes, G. Stasińska, A. Mateus, and N. Vale Asari, "A comprehensive classification of galaxies in the Sloan Digital Sky Survey: how to tell true from fake AGN?" Monthly Notices of the Royal Astronomical Society, vol. 413, no. 3, pp. 1687-1699, 2011.

[25] C. Kehrig, A. Monreal-Ibero, P. Papaderos et al., "The ionized gas in the CALIFA early-type galaxies: I. Mapping two representative cases: NGC 6762 and NGC 5966," Astronomy \& Astrophysics, vol. 540, article A11, 2012.

[26] G. Kauffmann, T. M. Heckman, C. Tremonti et al., "The host galaxies of active galactic nuclei," Monthly Notices of the Royal Astronomical Society, vol. 346, no. 4, pp. 1055-1077, 2003.

[27] L. J. Kewley, M. A. Dopita, R. S. Sutherland, C. A. Heisler, and J. Trevena, "Theoretical modeling of starburst galaxies," Astrophysical Journal Letters, vol. 556, no. 1, pp. 121-140, 2001.

[28] L. J. Kewley, B. Groves, G. Kauffmann, and T. Heckman, "The host galaxies and classification of active galactic nuclei," Monthly Notices of the Royal Astronomical Society, vol. 372, no. 3, pp. 961-976, 2006.

[29] R. C. Kennicutt Jr., W. C. Keel, and C. A. Blaha, "A comparison of the physical conditions in nuclear, hotspot, and disk H II regions," Astronomical Journal, vol. 97, pp. 1022-1035, 1989.

[30] L. C. Ho, A. V. Filippenko, and W. L. W. Sargent, "Properties of $\mathrm{H} 1$ regions in the centers of nearby galaxies," Astrophysical Journal Letters, vol. 487, no. 2, pp. 579-590, 1997.

[31] M. G. Allen, B. A. Groves, M. A. Dopita, R. S. Sutherland, and L. J. Kewley, "The mappings III library of fast radiative shock models," The Astrophysical Journal Supplement Series, vol. 178, no. 1, pp. 20-55, 2008.

[32] E. M. Levesque, L. J. Kewley, and K. L. Larson, “Theoretical modeling of star-forming galaxies. I. Emission-line diagnostic grids for local and low-metallicity galaxies," Astronomical Journal, vol. 139, no. 2, pp. 712-727, 2010.

[33] B. A. Groves, M. A. Dopita, and R. S. Sutherland, "Dusty, radiation pressure-dominated photoionization. II multiwavelength emission line diagnostics for narrow-line regions," Astrophysical Journal Supplement Series, vol. 153, no. 1, pp. 75-91, 2004.

[34] R. Cid Fernandes, G. Stasińska, M. S. Schlickmann et al., "Alternative diagnostic diagrams and the "forgotten" population of weak line galaxies in the SDSS," Monthly Notices of the Royal Astronomical Society, vol. 403, no. 2, pp. 1036-1053, 2010. 
[35] S. F. Sanchez, F. F. Rosales-Ortega, B. Jungwiert et al., "Massmetallicity relation explored with CALIFA. I. Is there a dependence on the star-formation rate?" Astronomy \& Astrophysics, vol. 554, article A58, 8 pages, 2013.

[36] P. Papaderos, J. M. Gomes, J. M. Vilchez et al., "Nebular emission and the Lyman continuum photon escape fraction in CALIFA early-type galaxies," Astronomy \& Astrophysics, vol. 555, article L1, 5 pages, 2013.

[37] R. C. Kennicutt Jr., "The global schmidt law in star-forming galaxies," Astrophysical Journal Letters, vol. 498, no. 2, pp. 541$552,1998$.

[38] M. A. Dopita, L. J. Kewley, C. A. Heisler, and R. S. Sutherland, "A theoretical recalibration of the extragalactic H II region sequence," Astrophysical Journal Letters, vol. 542, no. 1, pp. 224234, 2000.

[39] M. Fioc and B. Rocca-Volmerange, "PEGASE: a UV to NIR spectral evolution model of galaxies: application to the calibration of bright galaxy counts," Astronomy \& Astrophysics, vol. 326, no. 3, pp. 950-962, 1997.

[40] R. Terlevich, S. Silich, D. Rosa-González, and E. Terlevich, "How old are H II galaxies?" Monthly Notices of the Royal Astronomical Society, vol. 348, no. 4, pp. 1191-1196, 2004.

[41] S. F. Sánchez, F. F. Rosales-Ortega, R. C. Kennicutt Jr. et al., "PPAK wide-field integral field spectroscopy of NGC 628-I. The largest spectroscopic mosaic on a single galaxy," Monthly Notices of the Royal Astronomical Society, vol. 410, no. 1, pp. 313340, 2011.

[42] F. F. Rosales-Ortega, A. I. Díaz, R. C. Kennicutt Jr., and S. F. Sánchez, "PPAK wide-field Integral field spectroscopy of NGC 628-II. Emission line abundance analysis," Monthly Notices of the Royal Astronomical Society, vol. 415, no. 3, pp. 2439-2474, 2011.

[43] E. Mármol-Queraltó, S. F. Sánchez, R. A. Marino et al., "Integral field spectroscopy of a sample of nearby galaxies," Astronomy \& Astrophysics, vol. 534, article A8, 17 pages, 2011.

[44] K. Viironen, S. F. Sánchez, E. Marmol-Queraltó et al., "Spatially resolved properties of the grand-design spiral galaxy UGC 9837: a case for high-redshift 2-D observations," Astronomy \& Astrophysics, vol. 538, article A144, 2012.

[45] F. F. Rosales-Ortega, S. F. Sánchez, J. Iglesias-Páramo et al., "A new scaling relation for $\mathrm{H}$ II regions in spiral galaxies: unveiling the true nature of the mass-metallicity relation," Astrophysical Journal, vol. 756, no. 2, p. L31, 2012.

[46] I. Trujillo, G. Rudnick, H.-W. Rix et al., "The luminosity-size and mass-size relations of galaxies out to $z \sim 31$," Astrophysical Journal Letters, vol. 604, no. 2, pp. 521-533, 2004.

[47] M. Barden, H. W. Rix, R. S. Somerville et al., "GEMS: the surface brightness and surface mass density evolution of disk galaxies," Astrophysical Journal, vol. 635, no. 2, pp. 959-981, 2005.

[48] I. Trujillo, N. M. Förster Schreiber, G. Rudnick et al., “The size evolution of galaxies since $z \sim 3$ : combining SDSS, GEMS, and FIRES," Astrophysical Journal Letters, vol. 650, no. 1, pp. 18-41, 2006.

[49] J. C. Muñoz-Mateos, A. G. de Paz, S. Boissier et al., "Specific star formation rate profiles in nearby spiral galaxies: quantifying the inside-out formation of disks," Astrophysical Journal Letters, vol. 658, no. 2, pp. 1006-1026, 2007.

[50] L. A. MacArthur, J. J. González, and S. Courteau, "Stellar population and kinematic profiles in spiral bulges and discs: population synthesis of integrated spectra," Monthly Notices of the Royal Astronomical Society, vol. 395, no. 1, pp. 28-63, 2009.
[51] P. Sánchez-Blázquez, P. Ocvirk, B. K. Gibson, I. Pérez, and R. F. Peletier, "Star formation history of barred disc galaxies," Monthly Notices of the Royal Astronomical Society, vol. 415, no. 1, pp. 709-731, 2011.

[52] E. Pérez, R. Cid Fernandes, R. M. González Delgado et al., "The evolution of galaxies resolved in space and time: a view of inside-out growth from the califa survey," Astrophysical Journal, vol. 764, no. 1, p. L1, 2013.

[53] R. M. González Delgado, E. Pérez, R. Cid Fernandes et al., "The evolution of galaxies resolved in space and time: a view of inside-out growth from the CALIFA survey," Astrophysical Journal Letters, vol. 764, no. 1, article L1, 6 pages, 2013.

[54] S. Boissier and N. Prantzos, "Chemo-spectrophotometric evolution of spiral galaxies-I. The model and the Milky Way," Monthly Notices of the Royal Astronomical Society, vol. 307, no. 4, pp. 857-876, 1999.

[55] S. Boissier and N. Prantzos, "Chemo-spectrophotometric evolution of spiral galaxies-II. Main properties of present-day disc galaxies," Monthly Notices of the Royal Astronomical Society, vol. 312, no. 2, pp. 398-416, 2000.

[56] F. Bresolin, E. Ryan-Weber, R. C. Kennicutt Jr., and Q. Goddard, "The flat oxygen abundance gradient in the extended disk of M83," Astrophysical Journal, vol. 695, no. 1, pp. 580-595, 2009.

[57] P. Yoachim, R. Roškar, and V. P. Debattista, "Integral field unit spectroscopy of the stellar disk truncation region of NGC 6155," Astrophysical Journal Letters, vol. 716, no. 1, pp. L4-L8, 2010.

[58] R. A. Marino, A. Gil de Paz, A. Castillo-Morales et al., "Integral field spectroscopy and multi-wavelength imaging of the nearby spiral galaxy NGC $5668^{*}$ : an unusual flattening in metallicity gradient," Astrophysical Journal, vol. 754, no. 1, p. 61, 2012.

[59] F. Bresolin, R. C. Kennicutt Jr., and E. Ryan-Weber, "Gas metallicities in the extended disks of NGC 1512 and NGC 3621. Chemical signatures of metal mixing or enriched gas accretion?" Astrophysical Journal, vol. 750, no. 2, article 122, 2012.

[60] A. Gil de Paz, B. F. Madore, S. Boissier et al., "Discovery of an extended ultraviolet disk in the nearby galaxy NGC 4625," Astrophysical Journal, vol. 627, no. 1, pp. L29-L32, 2005.

[61] D. A. Thilker, L. Bianchi, G. Meurer et al., "A search for extended ultraviolet disk (XUV-disk) galaxies in the local universe," Astrophysical Journal Supplement Series, vol. 173, no. 2, pp. 538571, 2007.

[62] M. Vlajić, J. Bland-Hawthorn, and K. C. Freeman, "The abundance gradient in the extremely faint outer disk of NGC 300," Astrophysical Journal, vol. 697, no. 1, p. 361, 2009.

[63] C. ESteban, L. Carigi, M. V. F. Copetti et al., "NGC 2579 and the carbon and oxygen abundance gradients beyond the solar circle," Monthly Notices of the Royal Astronomical Society, vol. 433, no. 1, pp. 382-393, 2013.

[64] B. Husemann, K. Jahnke, S. F. Sánchez et al., "CALIFA, the Calar Alto Legacy Integral Field Area survey. II. First public data release," Astronomy \& Astrophysics, vol. 549, article A87, 25 pages, 2013.

[65] M. A. Lara-López, J. Cepa, A. Bongiovanni et al., "A fundamental plane for field star-forming galaxies," Astronomy \& Astrophysics, vol. 521, article L53, 5 pages, 2010.

[66] F. Mannucci, G. Cresci, R. Maiolino, A. Marconi, and A. Gnerucci, "A fundamental relation between mass, star formation rate and metallicity in local and high-redshift galaxies," Monthly Notices of the Royal Astronomical Society, vol. 408, no. 4, pp. 2115-2127, 2010. 
[67] S. F. Sanchez, F. F. Rosales-Ortega, J. Iglesias-Paramo et al., "A characteristic oxygen abundance gradient in galaxy disks unveiled with CALIFA," http://arxiv.org/abs/1311.7052.

[68] T. Tsujimoto, J. Bland-Hawthorn, and K. C. Freeman, "Evidence of early enrichment of the galactic disk by large-scale winds," Publications of the Astronomical Society of Japan, vol. 62, no. 2, pp. 447-456, 2010.

[69] F. Matteucci and P. Francois, "Galactic chemical evolutionabundance gradients of individual elements," Monthly Notices of the Royal Astronomical Society, vol. 239, pp. 885-904, 1989.

[70] B. E. J. Pagel and B. E. Patchett, "Metal abundances in nearby stars and the chemical history of the solar neighborhood," Monthly Notices of the Royal Astronomical Society, vol. 172, pp. 13-40, 1975.

[71] B. K. Gibson, Y. Fenner, A. Renda, D. Kawata, and H.-C. Lee, "Galactic chemical evolution," Publications of the Astronomical Society of Australia, vol. 20, no. 4, pp. 401-415, 2003.

[72] R. B. Larson, "Effects of supernovae on the early evolution of galaxies," Monthly Notices of the Royal Astronomical Society, vol. 169, pp. 229-246, 1974.

[73] J. Silk, "Dissipative processes in galaxy formation," Proceedings of the National Academy of Sciences of the United States of America, vol. 90, no. 11, pp. 4835-4839, 1993.

[74] S. Veilleux, G. Cecil, and J. Bland-Hawthorn, "Galactic winds," Annual Review of Astronomy and Astrophysics, vol. 43, pp. 769826, 2005.

[75] A. E. Shapley, C. C. Steidel, M. Pettini, and K. L. Adelberger, "Rest-frame ultraviolet spectra of $z \sim 3$ lyman break galaxies," Astrophysical Journal Letters, vol. 588, no. 1, pp. 65-89, 2003.

[76] B. J. Weiner, A. L. Coil, J. X. Prochaska et al., "Ubiquitous outflows in deep2 spectra of star-forming galaxies at $z=1.4$," Astrophysical Journal, vol. 692, no. 1, p. 187, 2009.

[77] A. Dekel, A. Zolotov, D. Tweed, M. Cacciato, D. Ceverino, and J. R. Primack, "Toy models for galaxy formation versus simulations," Monthly Notices of the Royal Astronomical Society, vol. 435, pp. 999-1019, 2013.

[78] S. J. Lilly, C. M. Carollo, A. Pipino, A. Renzini, and Y. Peng, "Gas regulation of galaxies: the evolution of the cosmic specific star formation rate, the metallicity-mass-star-formation rate relation, and the stellar content of halos," Astrophysical Journal, vol. 772, no. 2, p. 119, 2013.

[79] L. J. Kewley, D. Rupke, H. Jabran Zahid, M. J. Geller, and E. J. Barton, "Metallicity gradients and gas flows in galaxy pairs," Astrophysical Journal Letters, vol. 721, no. 1, pp. L48-L52, 2010.

[80] J. A. Rich, P. Torrey, L. J. Kewley, M. A. Dopita, and D. S. N. Rupke, "An integral field study of abundance gradients in nearby luminous infrared galaxies," Astrophysical Journal, vol. 753, no. 1, p. 5, 2012. 

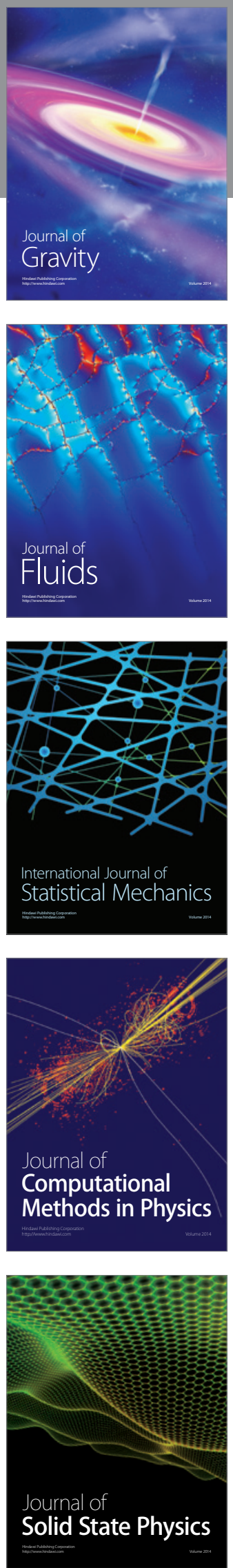

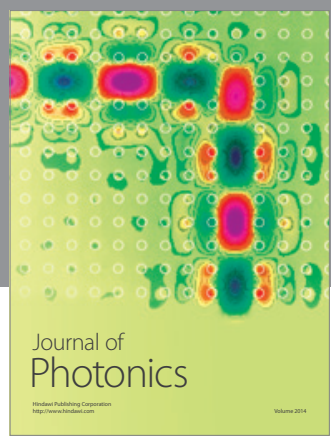

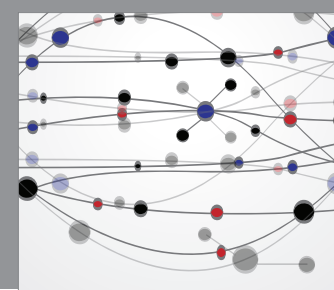

The Scientific World Journal

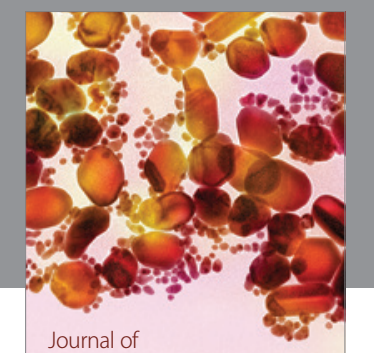

Soft Matter
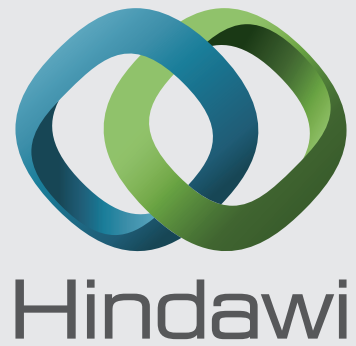

Submit your manuscripts at

http://www.hindawi.com
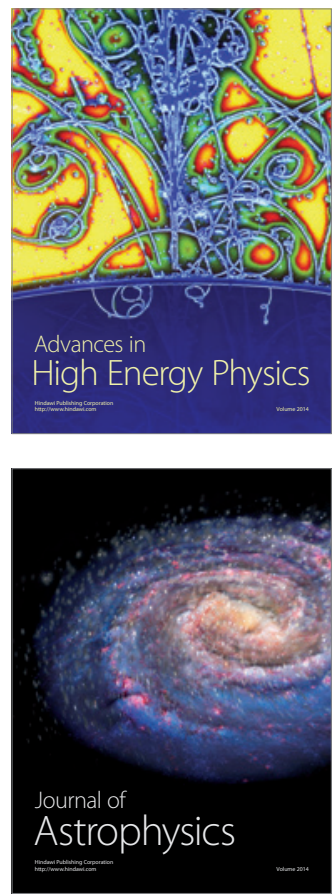
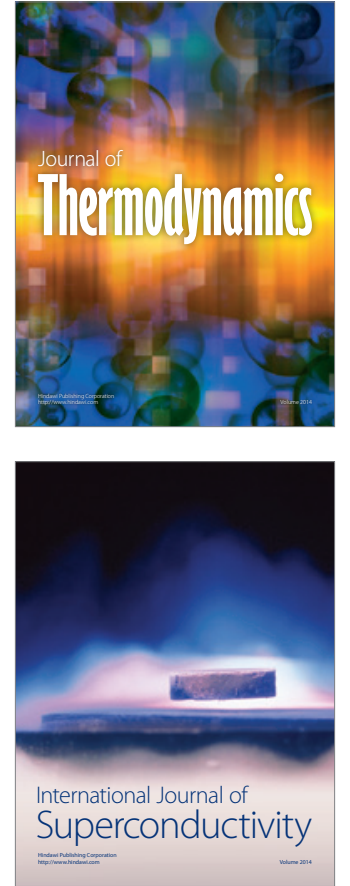
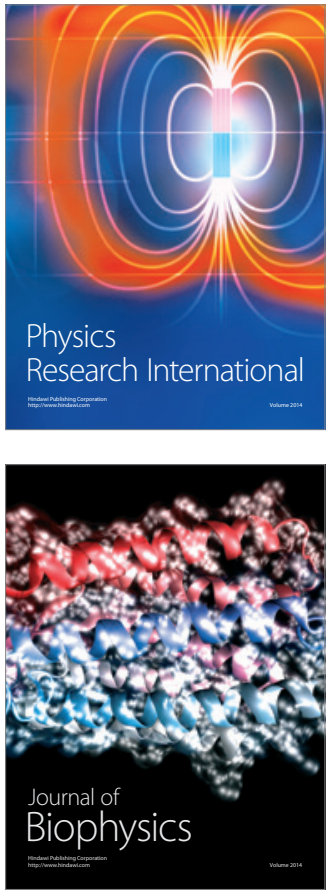
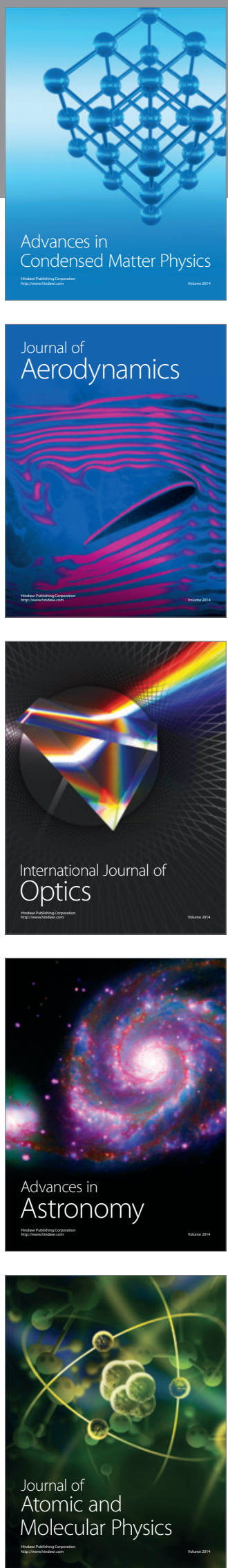